Article

\title{
Minimization of Losses in Smart Grids Using Coordinated Voltage Control
}

\section{Marko Kolenc *, Igor Papič and Boštjan Blažič}

Faculty of Electrical Engineering, University of Ljubljana, Tržaška 25, 1000 Ljubljana, Slovenia; E-Mails: igor.papic@fe.uni-lj.si (I.P.); bostjan.blazic@fe.uni-lj.si (B.B.)

* Author to whom correspondence should be addressed; E-Mail: marko.kolenc@fe.uni-lj.si; Tel.: +386-1-4768-901; Fax: +386-1-4768-289.

Received: 4 July 2012; in revised form: 21 August 2012 / Accepted: 19 September 2012 /

Published: 27 September 2012

\begin{abstract}
This article deals with the influence of distributed generation (DG) on distribution line losses with respect to voltage profile. The article focuses on the development of a control strategy to minimize the grid losses and assure fairness regarding reactive power contributions. As retail customers typically have no choice where they are located along a feeder, it seems unfair that only some of them bear all the burden and responsibility for the voltage rise. On the basis of new technologies, which are capable of fast communication and data processing, a new control system has been proposed that combines classical centralized and local control. The heart of the control system is a load-flow algorithm, which estimates the voltage drop using a modeled network. Different control solutions were evaluated by means of computer simulations. The simulated network is an actual Slovenian medium-voltage distribution network which covers a large area with diverse feeders and thus gives relatively general results.
\end{abstract}

Keywords: Smart Grid; distributed generation; voltage control; minimization of losses; reactive power flow

\section{Introduction}

High penetration levels of DG on an electrical distribution system present several challenges and opportunities for distribution utilities [1]. Their main concern is to maintain network voltage between tight limits, which is essential for correct operation of customer loads [2]. 
Classical control with On-Load Tap Changer (OLTC) is mostly based on only one substation voltage measurement point [3]. Such a scheme is robust and performs well under most operating conditions, but the majority of distribution networks are generally not designed for the connection of DG and they may fail if DG sources are connected in the network as they may cause a voltage rise on a particular feeder [2]. In order to introduce DG into the distribution grid, new control schemes have to be developed.

DG generation usually worked at a constant power factor $(\cos \varphi=1)$ and did not provide any ancillary services to the network; the voltage control, as previously mentioned, was carried out only by OLTC. Nowadays, many countries such as Slovenia have set up rules for DG to use static characteristic $Q(U)$ for the contribution with the local voltage control [4]. As shown in Figure 1, these characteristics determine, on the basis of current connection voltage, a specified $\operatorname{tg} \varphi$ operating point. $\operatorname{Tg} \varphi$ is the ratio between generator's reactive and active power. The level of $\operatorname{tg} \varphi$ determines the amount of reactive power dispatched, compared to currently produced active power. This relation can be written as:

$$
\operatorname{tg} \varphi=\frac{Q_{D G}}{P_{D G}}
$$

The product of $\operatorname{tg} \varphi$ and currently produced active power determines the amount of reactive power each generator has to provide. This kind of control allows the reduction of voltage by consuming reactive power and increasing the voltage by injecting it. The reactive power control with static characteristics should only be applied when a certain voltage level is reached, otherwise reactive power could result in unnecessary network losses.

Figure 1. Example of static $Q(U)$ characteristic for DG at full power, with up to $250 \mathrm{~kW}$ installed capacity, prescribed in Slovenia [4]. Similar characteristics are prescribed for the loads connected at LV or directly at MV network.

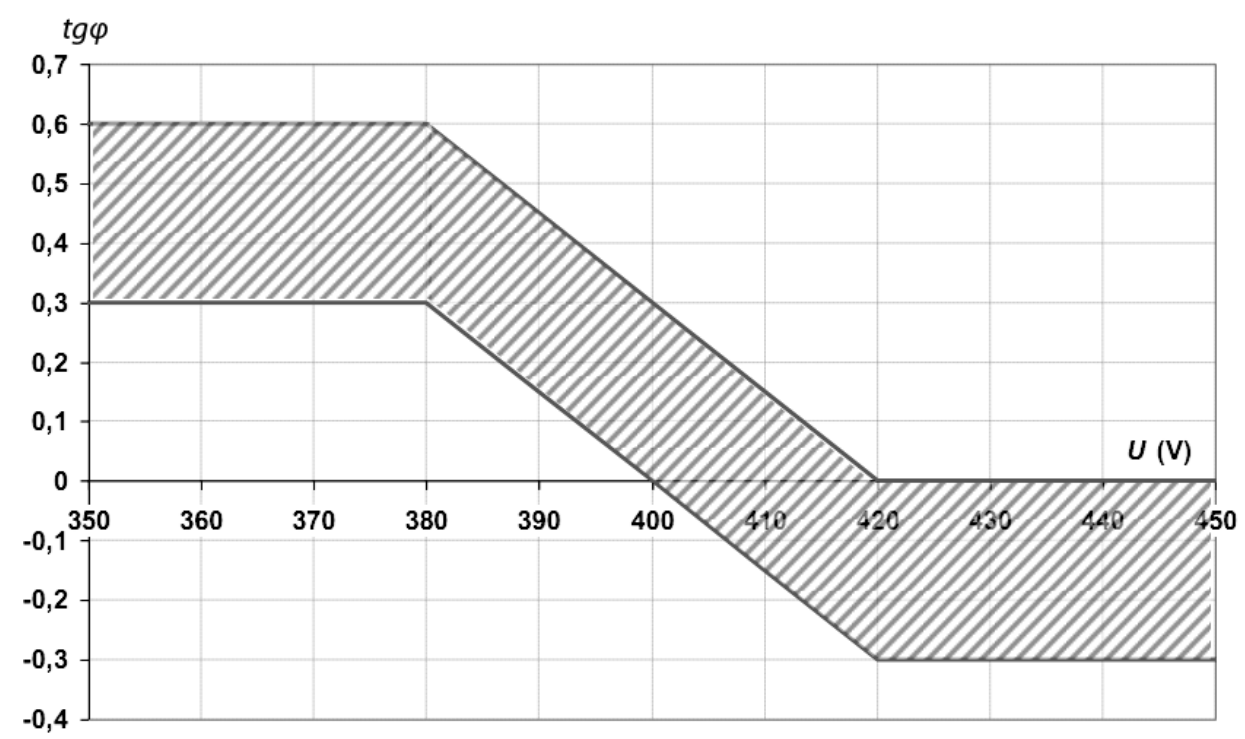

By using static characteristic, no communication link is required as the parameters are set during the installation of the DG. This approach has many disadvantages; although it is very effective in reducing the voltage rise, it does not enable fair contribution of reactive power. Dispatching reactive power 
imposes an additional burden on the DG inverters, which may lead to their reduced lifetime and an increased lifecycle cost [5]. If a customer lives far away from the substation, where voltage deviations are more frequent, the inverters will have to inject or consume more reactive power than for those located in areas where the voltage deviations are minor. Also their location may change depending on how the entire distribution system is configured. As retail customers typically have no choice where they are located along the feeder, it seems inappropriate that they be required to produce or consume a large amount of reactive power and hence, bear all the burden and responsibility for the voltage rise along the entire feeder.

In this article a coordinated approach to upgrade previously described controls is presented, which is based on a time-dependent power factor. By using time-dependent DG set-points new control possibilities become possible. The heart of the control system is a load flow algorithm, which estimates the voltage drop using a modeled network. The load-flow algorithm is, on the basis of measurements and load forecast prediction, continuously running and searching for an optimum operating point. A simplified model of the proposed algorithm has been used in [6], wherein the authors have dealt with coordination of capacitor banks through the network to obtain an optimal switching scheme. Using voltage-profile estimation and load forecasting information, a load-flow iteration procedure has been implemented to search through all the possible change in voltage arrays to find the best solution for the next 24-h time horizon. A parallel between this kind of control and the tap changer switching mechanism can be drawn. In the article the above suggested idea has been upgraded by inclusion of local DG control and centralized OLTC control into one algorithm. The goal was to minimize the losses subject to a fair contribution of all DG with respect to voltage limits.

This article is organized as follows. The principle of a fair contribution of reactive power is presented in Section 2. Technical requirements, which are important for the proposed control mechanism, are presented in Section 3. Section 4 presents the control system principle. The simulated network is described in Section 5 and the simulation results are shown in Section 6. Finally, the conclusions are drawn in Section 7.

\section{Fair Contribution of Reactive Power}

To allocate the contribution of all DG reactive power more efficiently, solutions such as location-based static characteristics were investigated in [7]. This approach resulted in slightly better voltage profiles. The advantage of this approach is that no communication was required, apart from the one-time coordinated parameterization of the $Q(U)$ functions of each DG. However, such a solution is appropriate only for network configurations where the parameters are fixed at the installation; they are no longer suitable when the network is reinforced or extended.

In [8], the necessary reactive DG power set-point was sent to generators based on their effect on the voltage profile. A sensitivity factor was introduced, which describes how much voltage change is caused by a change in the injected or consumed power. This approach has a positive impact on the voltage profile and the dispatch of a more uniformly spread reactive power but it is in conflict with our aim to not penalize retail customers as they cannot change their electrical location along the feeder. However, this seems to be one possible solution to mitigate the voltage rise in the case of an emergency. 
Uniform $\operatorname{tg} \varphi$ can be seen as a tax system with a constant marginal tax rate in the business world. There is also an option to introduce a progressive taxation system, where entities with the same economic power pay the same amount of taxes, while the subjects with greater economic power should pay higher taxes. Progressive tax rate increases the tax base, resulting in an increase in tax rate. Fair taxation would be spread widely and evenly among all taxpayers [9].

Equations that represent the amount of required reactive power with a uniform and progressive "tax rate" can be written as:

$$
\begin{gathered}
Q_{g}=P_{g} \cdot \operatorname{tg} \varphi \\
Q_{g}=\left(P_{g}\right)^{x} \cdot \operatorname{tg} \varphi
\end{gathered}
$$

where $x$ represents a progressive tax rate with exponential dependence. The progressive tax rate can be also carried out with different tax classes. In that case, a proportional variable can be added to (2), which discretely changes its size based on the active power size [9].

As an option to ensure fairness, a uniform $\operatorname{tg} \varphi$ was used in further simulations presented in Section 6. To every feeder in the network, a uniform $\operatorname{tg} \varphi$ operating point was sent. Hence, the amount of reactive power is not based on their electrical location on the feeder, but only on their size. Their electrical location is limited only on one feeder as a whole unit.

\section{Technical Requirements}

At regular time intervals the operating point of DG is changed by the transmitted signal. Because the desired operating point is calculated based upon present values, an effective operation requires an accurate evaluation of the situation in the network, which could be difficult in some cases. However, R\&D projects all around the world show that the technologies are mature [10-12] and the premise of a time-dependent power factor and bidirectional dataflow is justified. For the success of the proposed coordinated control, most of the MV/LV transformer substations need to be covered with real-time measurements to obtain the information of the consumption and then calculate the load-flow. In areas where there are no measurements, typical daily load patterns can be presumed and normalized to the size of the complex.

As the data bandwidth requirements for DG control is relatively low; response times are in the range of seconds $[13,14]$, the DG's have to be able to produce or consume the needed reactive power. In [1], overrating of inverters by $10 \%$ is considered, which seems reasonable because inverters are available in discrete sizes and they are likely to be oversized. With respect to $10 \%$ overrating, available reactive power always reaches at least $46 \%$ of $P_{\text {MAX }}[15]$.

\subsection{Modeling of the Loads and Generators}

For the algorithm to work successfully, modeling of the loads has to be carefully considered. Different characteristics of loads connected to distribution feeders affect power losses significantly.

Loads in the simulated network described in Section 5 were modeled with a polynomial model [16]: 


$$
\begin{aligned}
& P=P_{0}\left[p_{1}\left(\frac{U}{U_{0}}\right)^{2}+p_{2}\left(\frac{U}{U_{0}}\right)+p_{3}\right] \\
& Q=Q_{0}\left[q_{1}\left(\frac{U}{U_{0}}\right)^{2}+q_{2}\left(\frac{U}{U_{0}}\right)+q_{3}\right]
\end{aligned}
$$

For an accurate evaluation of losses, connected loads on every feeder have to be assessed. Load modeling has been widely discussed in the literature. A detailed analysis can be found in [16].

Figure 2 shows how the amount of losses strongly depends on the coefficients $\alpha$ and $\beta$ when using an exponential load model:

$$
\begin{aligned}
& P=P_{0}\left(\frac{U}{U_{0}}\right)^{\alpha} \\
& Q=Q_{0}\left(\frac{U}{U_{0}}\right)^{\beta}
\end{aligned}
$$

Additionally, panel no. 1 in Figure 2 represents the losses with OLTC feeding voltage, which is lower compared to panel no. 2. It can be seen that in the case when the loads have constant impedance character, the losses are smaller at smaller voltages (lower tap position) and when the loads have the character of constant power, the losses are smaller at higher voltages (higher tap position).

Figure 2. Losses with respect to coefficients $\alpha$ and $\beta$ and OLTC feeding voltage.

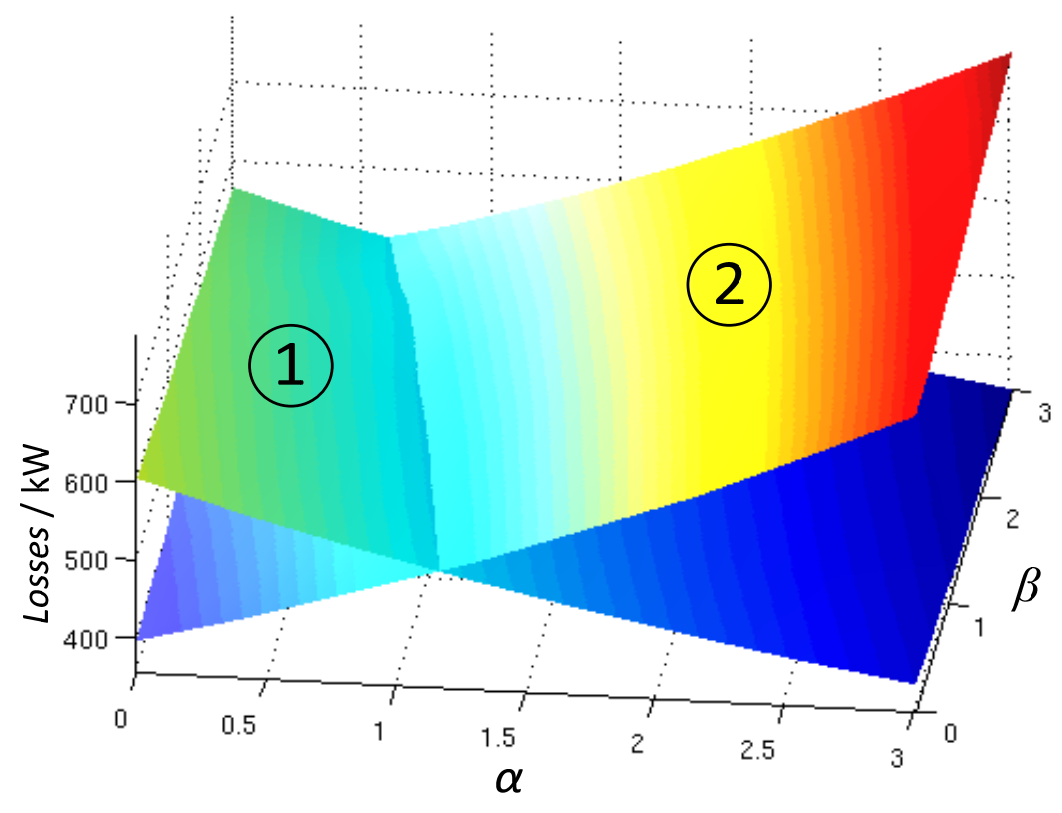

\subsection{Generation Forecast}

Forecast information is necessary for the management of electricity grids and also for solar energy trading [17]. For load consumption and generation from classical centralized power plants, forecasting has high accuracy and has been discussed in the literature very often. Electrical loads for HV/MV distribution substations can be predicted with about $1.5 \%$ accuracy for a 24-h forecast [18] and up to $5 \%$ for $\mathrm{MV} / \mathrm{LV}$ substations [19,20]. Larger fluctuations in the forecast of electricity production are 
present in small renewable energy sources, particularly in wind or photovoltaic power. An accurate forecast allows stable operation of the system without excessive energy reserves. Consequently, research on medium and long-term forecast methods has gained great importance both in academia and industry [21]. There are several such methods described in the literature based on artificial neural networks, fuzzy models, support vector regression etc. A good overview on the subject is provided in [18,21]. Typical wind power plants have an error up to $15 \%$ rated power for 36 hour horizont. An accurate prediction of the future state of the network plays an important role in the efficient management of the proposed algorithm when optimizing OLTC set-point and minimizing its operations. An accurate prediction of generation for about $10 \mathrm{~h}$ would be ideal for its optimal performance. In such cases, a range of errors around $10 \%$ or less is to be expected $[22,23]$. In addition, during evening and night h (6 PM-9 AM), the forecast can be even more accurate due to minimal solar radiation and thus, a small $\mathrm{PV}$ production.

\subsection{Limits and Objectives to Be Minimized}

The development of a good voltage control system must take into account several parameters. The OLTC's tap position has to be controlled; DG reactive power and in some cases even active power has to be controlled; OLTC can be connected to many diverse feeders whose power consumption or production can differ widely; also network reconfiguration can cause significant differences in the network. Every time an optimal operation has to be ensured.

The main condition, which must be assured regardless of any other, states that the voltage at any point of the network shall not exceed statutory defined limits:

$$
0.95 \leq U \leq 1.05
$$

Taking into account that the voltage drop on a MV/LV transformer feeding loads and LV feeder can reach $5 \%$ and that the voltage control step is $1.33 \%$, the MV voltage limits were set to $+5 /-5 \%$. The voltage control technique therefore kept the MV within these limits, and consequently that should enable the maintenance of LV within the defined range.

Using coordinated control algorithms, the following conditions can be minimized:

$$
\begin{gathered}
\text { losses } \rightarrow \text { min } \\
D G \text { reactive power generation } \rightarrow \text { min } \\
\text { OLTC operations } \rightarrow \text { min }
\end{gathered}
$$

On assuming a fair distribution of reactive power from DG, these conditions can be guaranteed.

\section{Control System Design}

Figure 3 represents an active distribution network. The current information of consumption is broadcasted to the central control system and the calculated commands are then sent back to the grid. 
Figure 3. Active distribution network.

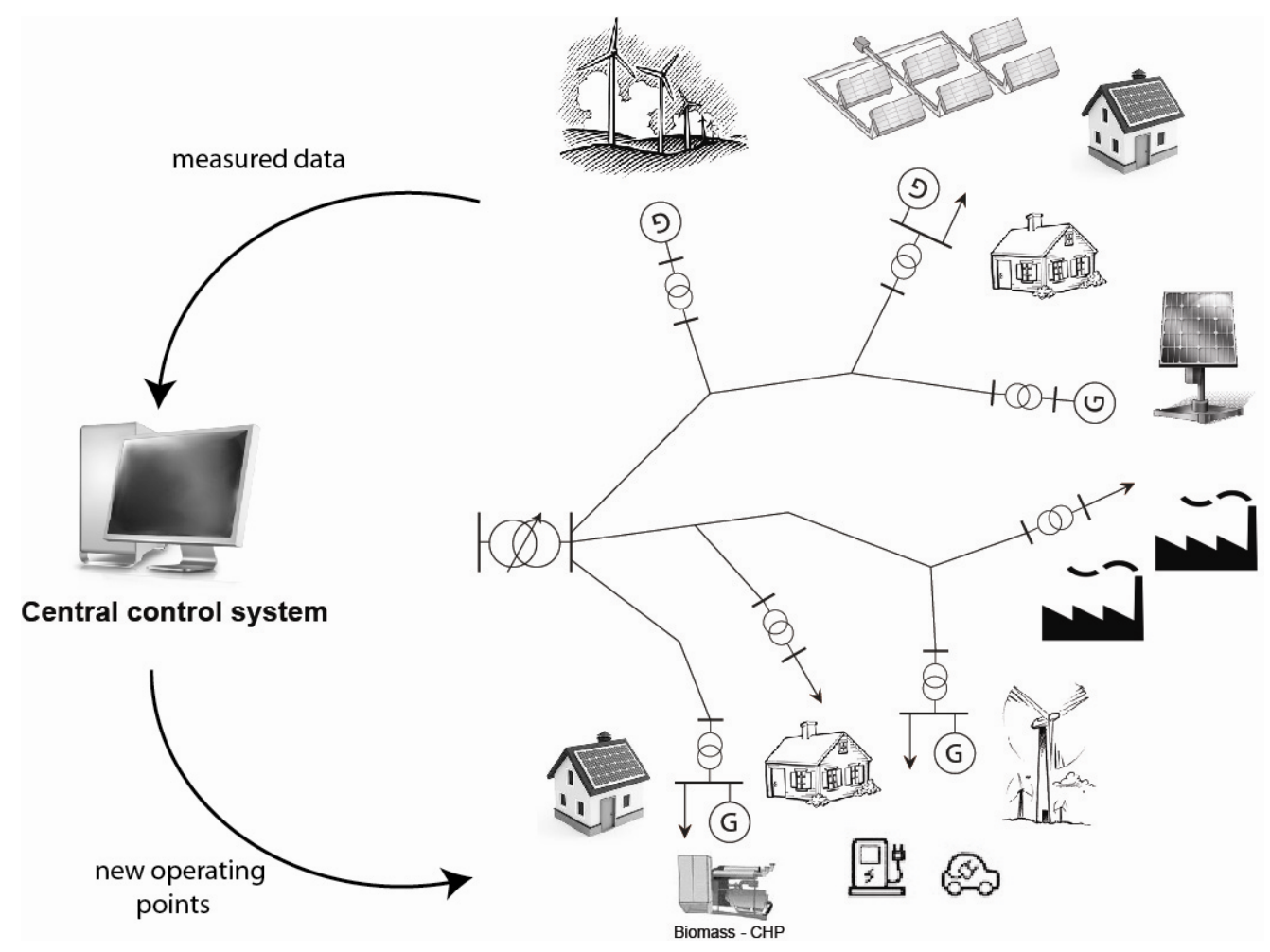

Current power $(P$ and $Q$ ) quantities are used as an input of load flow algorithm, which calculates the optimal $\operatorname{tg} \varphi$. Two main criteria (9) and (10) are taken into account. Subject to (9), $\operatorname{tg} \varphi$ is set to a specified value where the losses are minimal and subject to (10), $\operatorname{tg} \varphi$ should be assigned a minimum value as it presents ancillary services cost. However, searching for the best $\operatorname{tg} \varphi$ is subject to the voltage condition (8). Taking into account the statutory defined voltage conditions, different set-points for $\operatorname{tg} \varphi$ are given. Figure 4 shows an example of the desired $\operatorname{tg} \varphi$ of one feeder per day. The black line indicates the desired set-point of minimal losses. Because of the voltage limits in some cases during the day, $\operatorname{tg} \varphi$ has to withdraw this trajectory (blue line). Similarly, the green line represents $\operatorname{tg} \varphi$ when reactive power generation from DG is minimal with respect to voltage limits as $\operatorname{tg} \varphi=0$ could lie outside the feasible region.

Possessing different set-points arises the question which one is more appropriate to take. Besides line loss minimization, equipment operation ( $Q$ generation), which contributes to the aging of inverters, has to be financially evaluated. In other words, the ancillary services should also be payable. Regarding the financial aspect, an operating point of minimum cost can be chosen. This principle can be observed in Figure 5. By doing this, both the network utility and DG owners gain financial benefit. Taken for granted minimal allowable power factor, the chosen value is then sent to the generators. 
Figure 4. Reference $\operatorname{tg} \varphi$ tracking of one feeder.

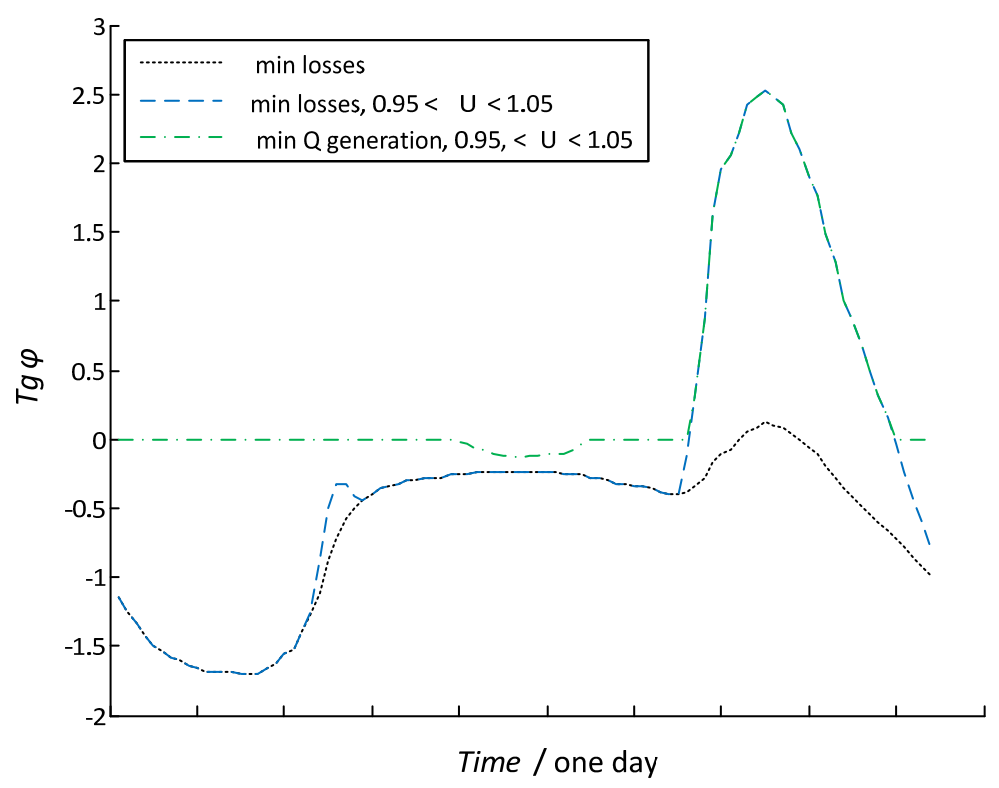

Figure 5. Principled graphical representation of the optimization problem.

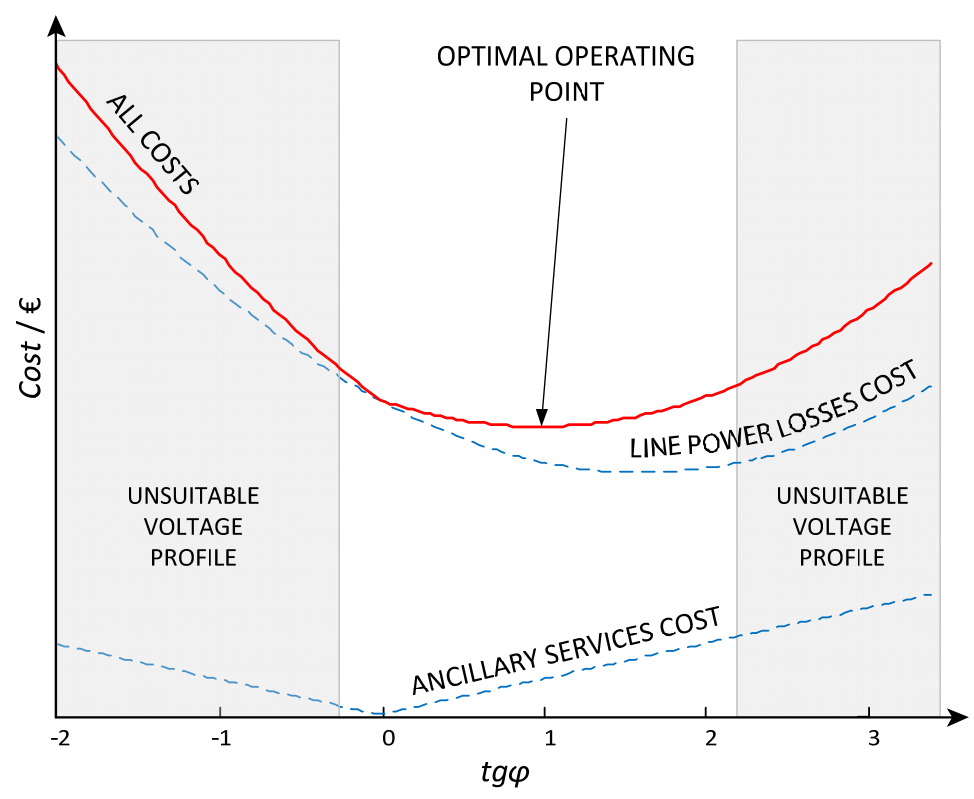

The flow chart of the algorithm is presented in Figure 6. Using power measurements from MV/LV substations and network topology (modeled network), a power-flow algorithm can be run to search for $\operatorname{tg} \varphi$ where the losses are minimal. The minimal and maximal possible tg $\varphi$ were set to \pm 3 to better illustrate the results but depending on the expected minimum this interval can be changed and power-flow operations reduced. Additionally, the search accuracy ( 0.01 steps $)$ can be changed. Once the optimal $\operatorname{tg} \varphi$ for each feeder is chosen, the power-flow is then run once again to check if the voltages are still within the limits. If not, corrections have to be made and the $\operatorname{tg} \varphi$ changed.

The algorithm does not only search for an optimal operating point of DG but also for an optimal OLTC set-point. Figure 7 shows an example of a one-day voltage profile of one feeder with a relatively high penetration of photovoltaic generators. As the algorithm calculated that the losses are at a minimal 
when the voltages are right above the permitted limit, which is usually true when the loads in the network express a constant impedance characteristic, the OLTC set-point was turned down as soon as possible. It can be clearly seen that in the search for minimal losses, the stress in tap-changing has increased greatly. Such a high number is not acceptable. Hence, the load forecast is implemented into the load-flow simulation program. The algorithm can determine when the next tap change operation will occur and if it is economically justified. On the basis of load forecast, simulations are carried out in the presence and absence of switching.

Figure 6. Algorithm flowchart.

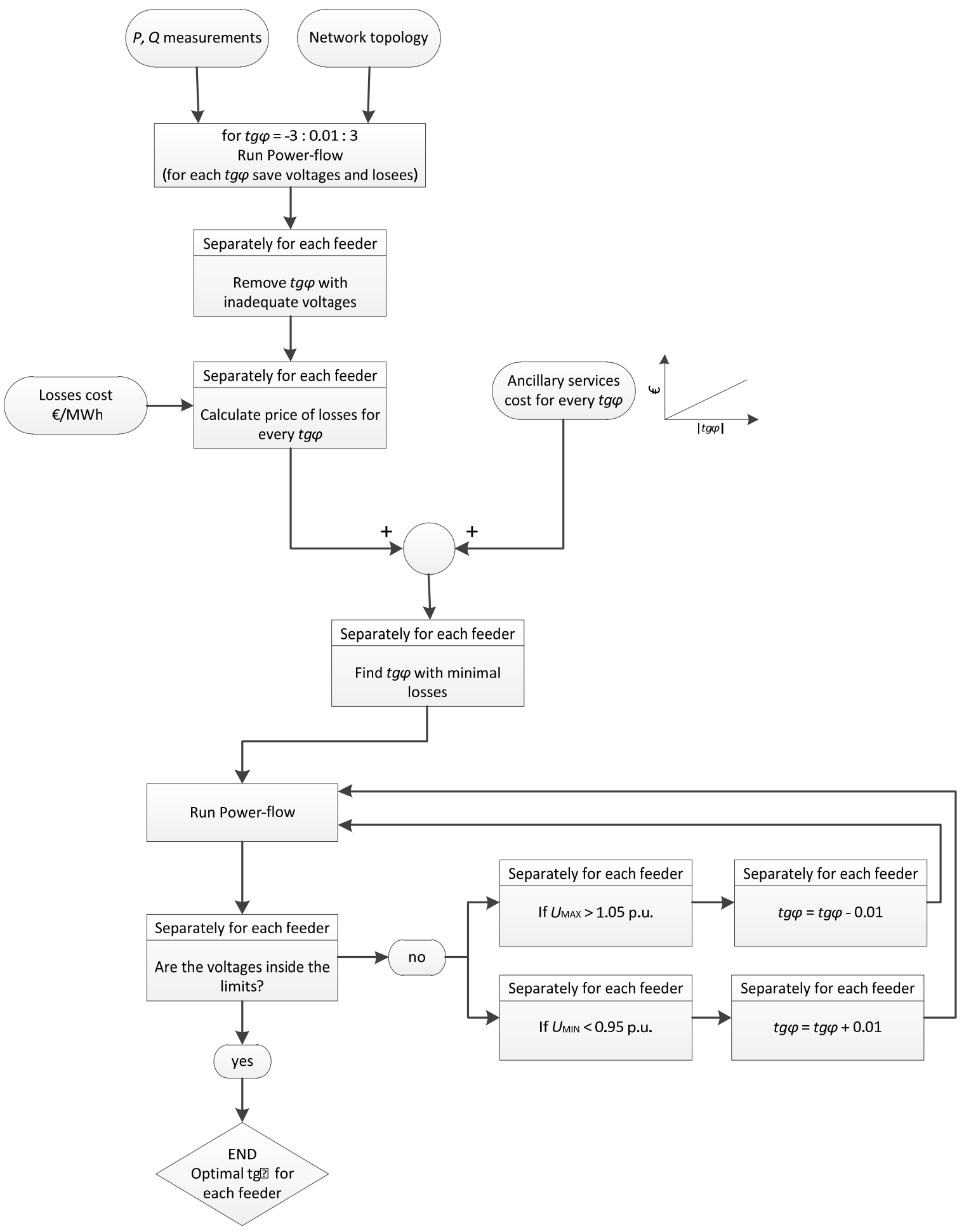


Figure 7 also shows a few examples of decision-making. Savings in energy losses are calculated for the period between two operations with the same voltage level. It should be highlighted that the cost of switching the tap position has to be evaluated. This allows the algorithm to decide if it is better to switch and reduce the losses or let the tap position remain in the same state.

Figure 7. One day voltage profile of MV/LV substations. The green dotted lines marked from 1 to 5 represent a few possible scenarios in which the decision to switch or not must be made. The algorithm generates simulations between two tap positions which have the same value.

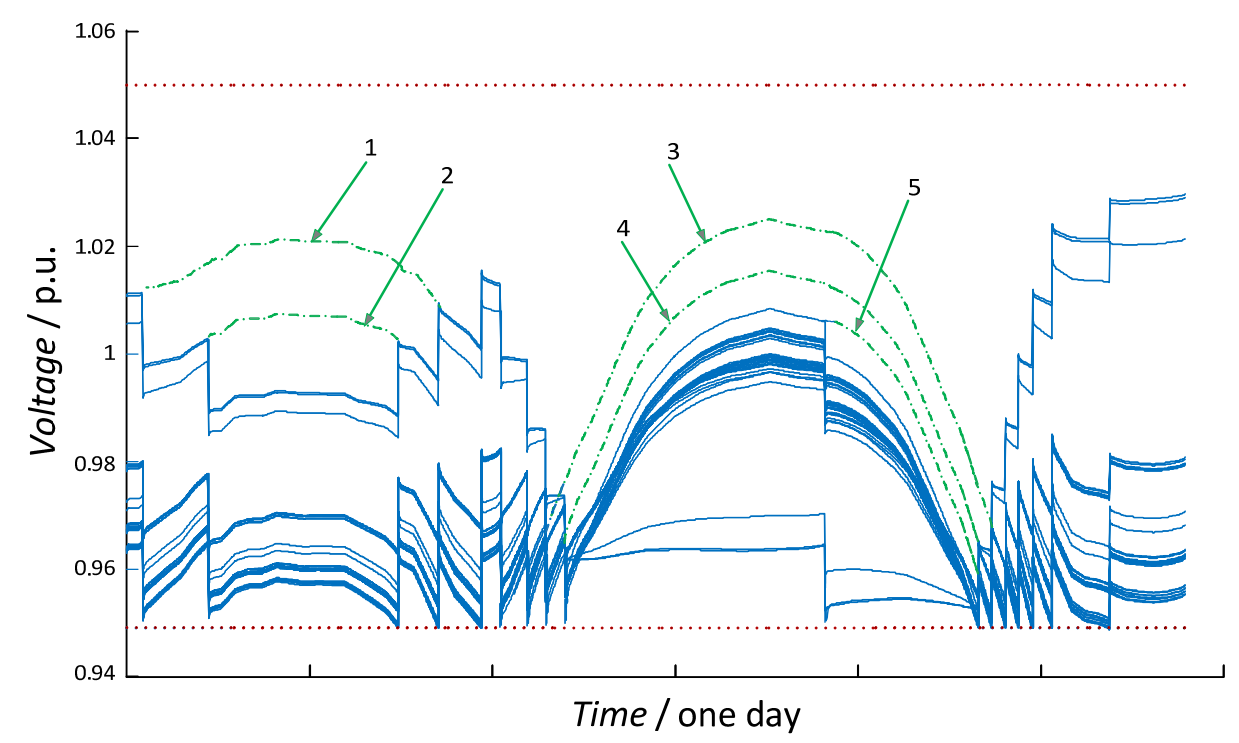

It must be noted that setting the optimal $\operatorname{tg} \varphi$ has a priority over the OLTC forecasting algorithm. This means that if the voltage profile is unsuitable due to inaccurate load forecasts under which OLTC was maintaining the tap position at the same state, OLTC will operate before $\operatorname{tg} \varphi$ can be correlated, although this is not a desired effect. This phenomenon occurs due to energy savings. The simulation results in Section 6 show that most of the savings fall on setting an optimal $\operatorname{tg} \varphi$.

\section{Simulated Network Description}

To illustrate some practical implications of the proposed voltage-control algorithm, the operation is demonstrated on a real $20 \mathrm{kV}$ medium-voltage Slovenian distribution network model. It covers a large area with 483 loads and diverse feeders and thus gives relatively general results. The network was modified by increasing the number of DG (especially photovoltaic and cogeneration power plants) as their growth in Slovenia is very fast.

The single-line diagram of the analyzed network is shown in Figure 8. The network was modeled in the DIgSILENT Power Factory simulation program and the voltage control algorithm in Matpower 4.1 [24]. The control maintains the voltages between the limits of 0.95 and 1.05 p.u.

The $20 \mathrm{kV}$ network is connected to the HV level at $110 \mathrm{kV}$ through a $120 \mathrm{MVA}$ transformer equipped with OLTC (control $\pm 12 \%$, step $1.33 \%$ ). Loads and generators were modeled as $R-X$ impedances at $20 \mathrm{kV}$ voltage level that are also voltage dependent (in the voltage range $80 \%-120 \%$ of the nominal value) and were modeled as composite loads consisting, in the first case, of $100 \%$ constant 
power and in the second case $60 \%$ constant impedance and $40 \%$ constant power. Based on the loading measurement data (10-minute intervals), few typical daily load patterns were developed (residential, commercial and industrial loads). One of the patterns is shown in Figure 9. For loads, a power factor $\cos \varphi \approx 0.95$ was presumed.

New operating points were calculated every minute of the simulation and sent to the generators. The maximal peak consumption is ca. $70 \mathrm{MW}$ (at 19:15) and maximal peak generation from DG is ca. $100 \mathrm{MW}$ (at 12:00). Depending on the size of each settlement with an MV/LV substation, represented as one load, an adequate size of the generation was determined. The maximum active power output of 167 generators was less than $0.1 \mathrm{MW}$, of 251 generators between 0.1 and $0.5 \mathrm{MW}$, of 40 generators between 0.5 and $1 \mathrm{MW}$ and of 7 generators more than $1 \mathrm{MW}$.

Figure 8. Large medium-voltage distribution system under study.

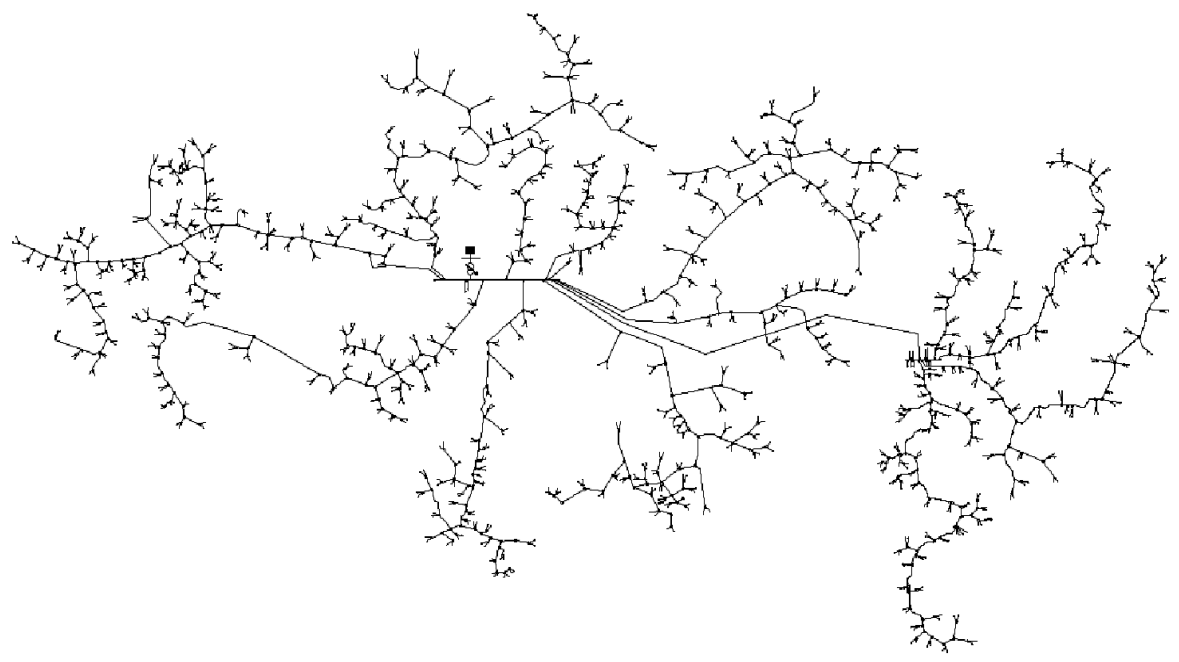

Figure 9. Typical LV substation daily load pattern for (a) residential and (b) industrial areas.
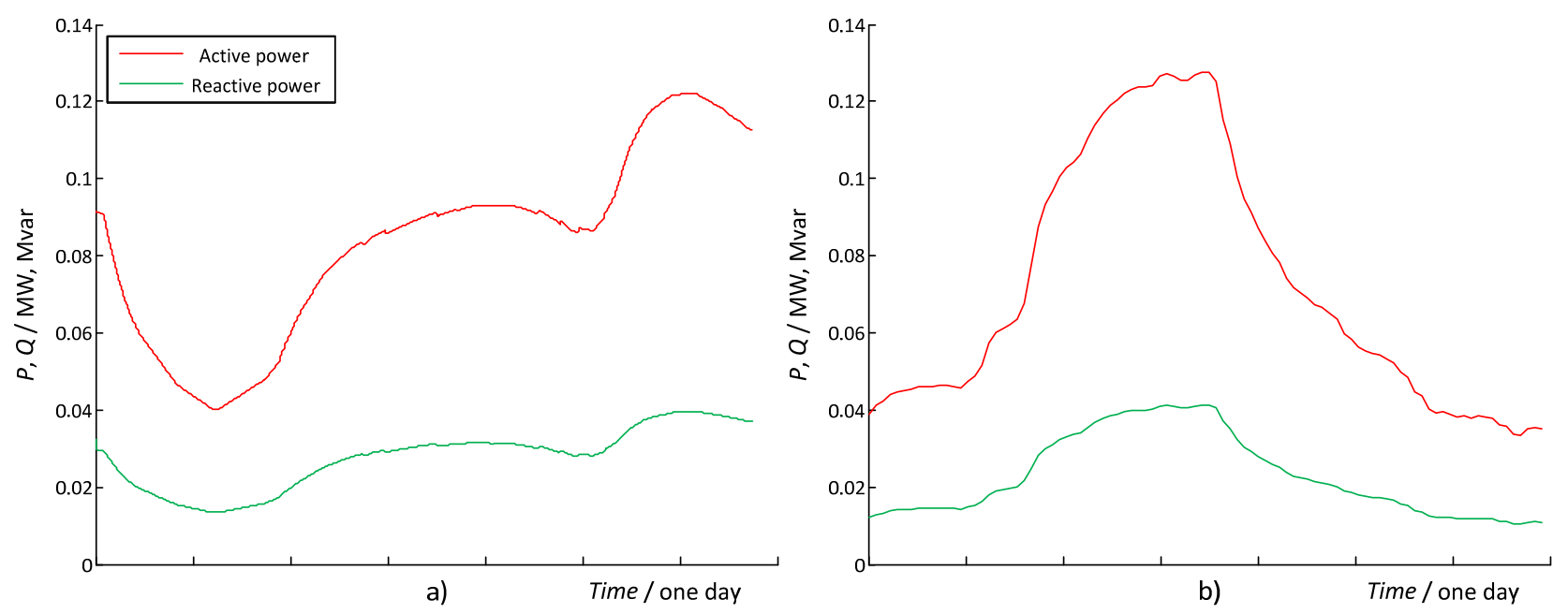

\section{Control System Performance Evaluation}

Simulation results for one day are presented in this section and compared between different controls. The simulations were carried out based on uniform $\operatorname{tg} \varphi$. Moreover, the impact of errors in modeling of loads described in Section 3 was also taken under consideration. 


\subsection{Static Methods}

Simulations of this type were carried out in a way that reactive power control and OLTC set-point control acted independently from each other. There is no need for communication; only local measurements are used for each type of control.

Figure 10(a) represents a voltage profile before the introduction of DG into the network. It is seen that 3-4 tap operations per day are needed to obtain a voltage profile within the prescribed limits. The line network losses are around 3\%. The DG was introduced to the network later.

Figure 10. One day voltage profile of MV/LV substations measured at the critical areas around the distribution system. (a) Voltage profile before the introduction of DG; (b) voltage profile with DG working with $\cos \varphi=1$; (c) voltage profile using static $Q(U)$ characteristic.
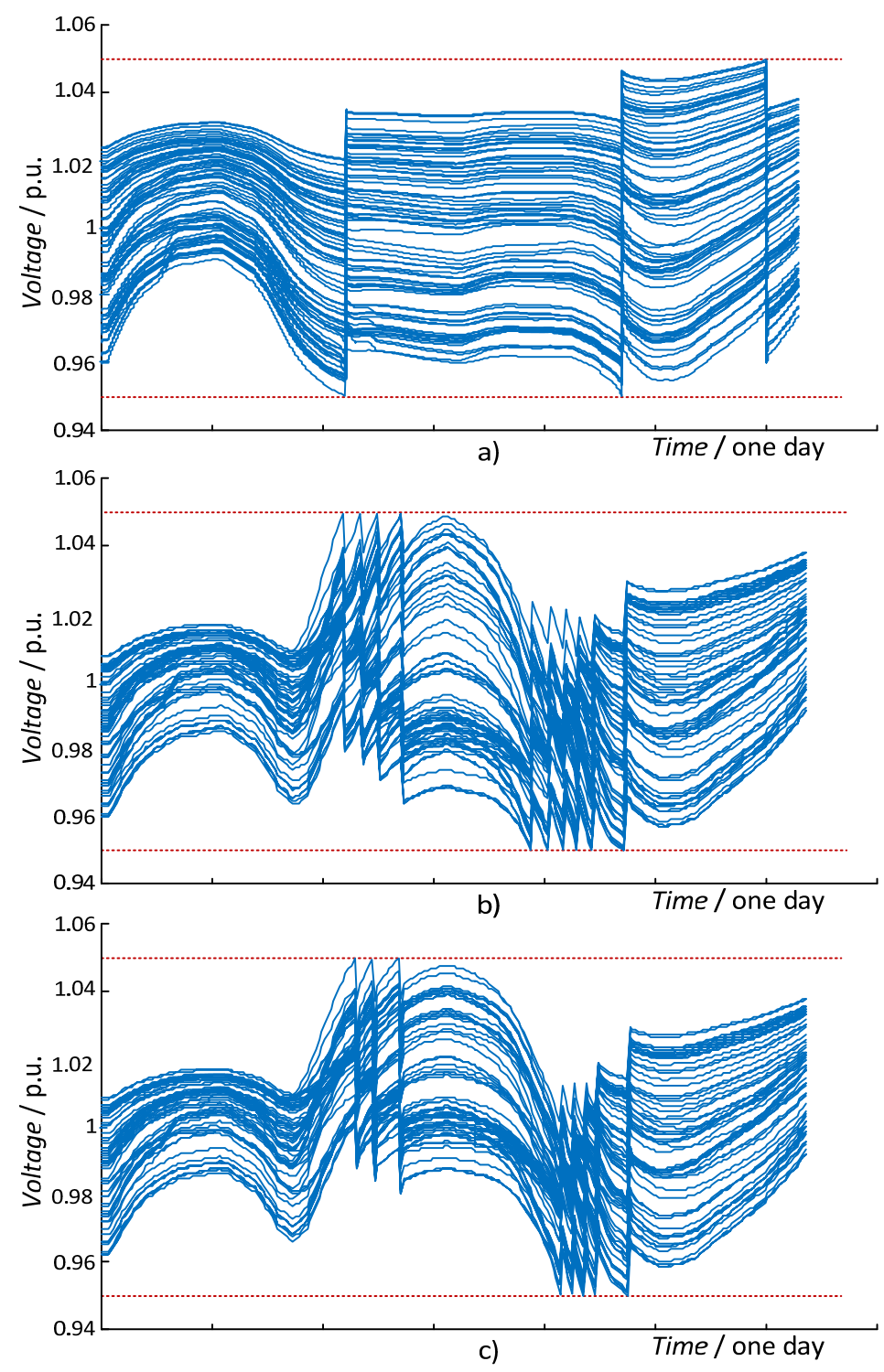

Figure 10(b) shows the results when DG does not assist in the voltage control and Figure 10(c) shows the results using the static $Q(U)$ characteristic. It is clearly seen that the second case is far more effective in reducing the voltage rise caused by injected DG active power, which means that the 
network can accept more DG. However, this action is performed even when the reduction of voltage rise is not necessary, thus contributing to higher network losses. Furthermore, the generators at the beginning of the feeder are low burdened with reactive power compared to generators at the end of the feeder, which are producing or consuming the majority of the reactive power as observed from Figure 11. Figure 12 additionally shows that the effect of the Sun during the day causes the reversal of the active power flow through the OLTC transformer.

Figure 11. $\operatorname{Tg} \varphi$ for four different generators in the same feeder. Generators have to produce different amounts of reactive power based on their location.

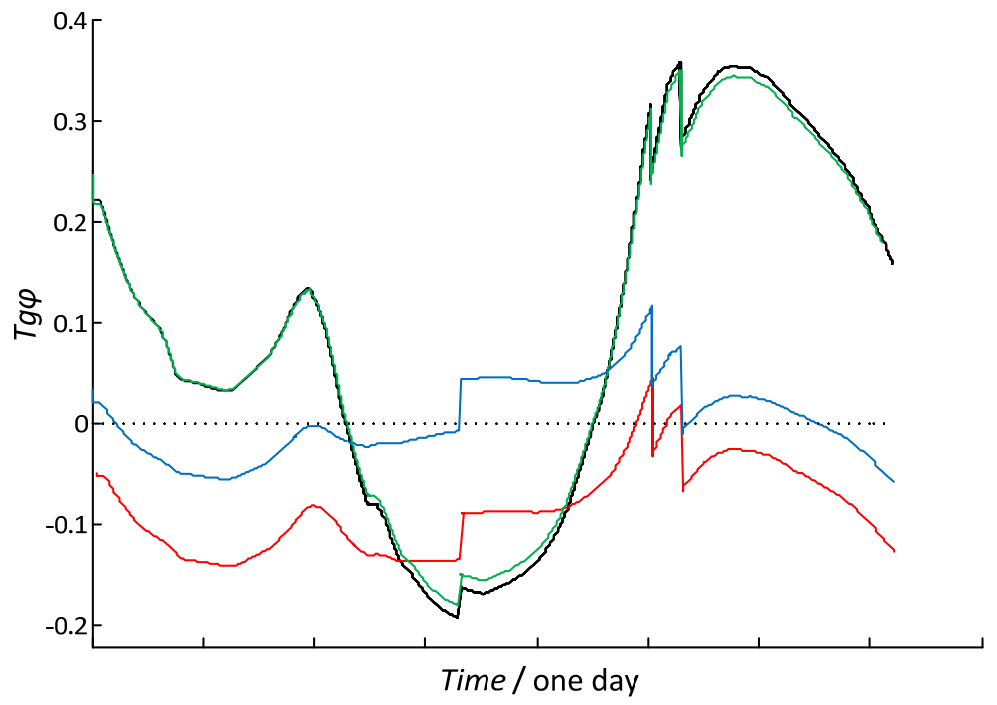

Figure 12. Power flow through the OLTC transformer. It can be seen that during peak $h$ of sunlight, the power flow is reversed.

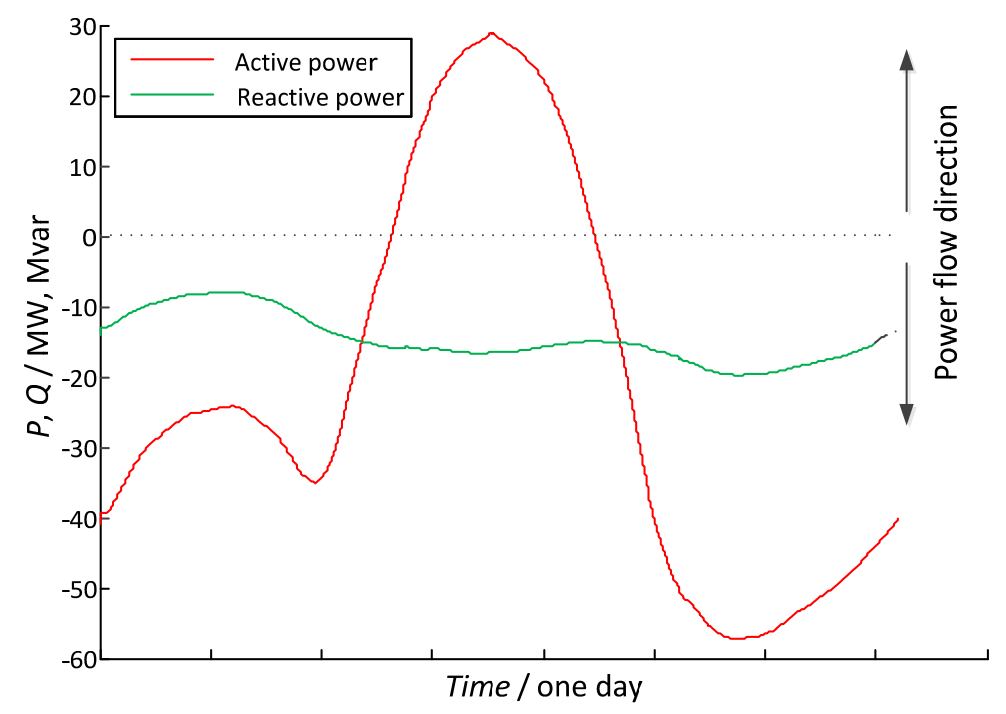

\subsection{Coordinated Control}

Firstly, simulations were carried out using only a time-dependent power factor. Figure 13(a) represents the voltage profile for a $\operatorname{tg} \varphi$ set-point with minimal losses. The operation of the whole algorithm is shown in Figure 13(b), which represents the voltage profile for a $\operatorname{tg} \varphi$ with minimal losses 
and an optimal OLTC set-point. The OLTC tap operations were unlimited and thus, with respect to a fair reactive power dispatch, minimal losses in the network were achieved.

\subsection{Results Evaluation}

In this Section, the proposed algorithm is validated by comparing the losses and tap-changer operations. The results obtained by using the loads with constant power voltage dependency are summarized in Table 1. Annual monetary savings are compared to static $Q(U)$ control. The price for electrical energy was assumed to be $50 € / \mathrm{MWh}$.

Figure 13. One-day voltage profile of $\mathrm{MV} / \mathrm{LV}$ substations measured at the critical areas around the distribution system using a time-dependent $\operatorname{tg} \varphi$. (a) Voltage profile for $\operatorname{tg} \varphi$ set-point with minimal losses; (b) voltage profile of the whole algorithm for $\operatorname{tg} \varphi$ with minimal losses and optimal OLTC set-point; (c) during the evening the tap-changer did not change its position and thus operations were reduced for two.

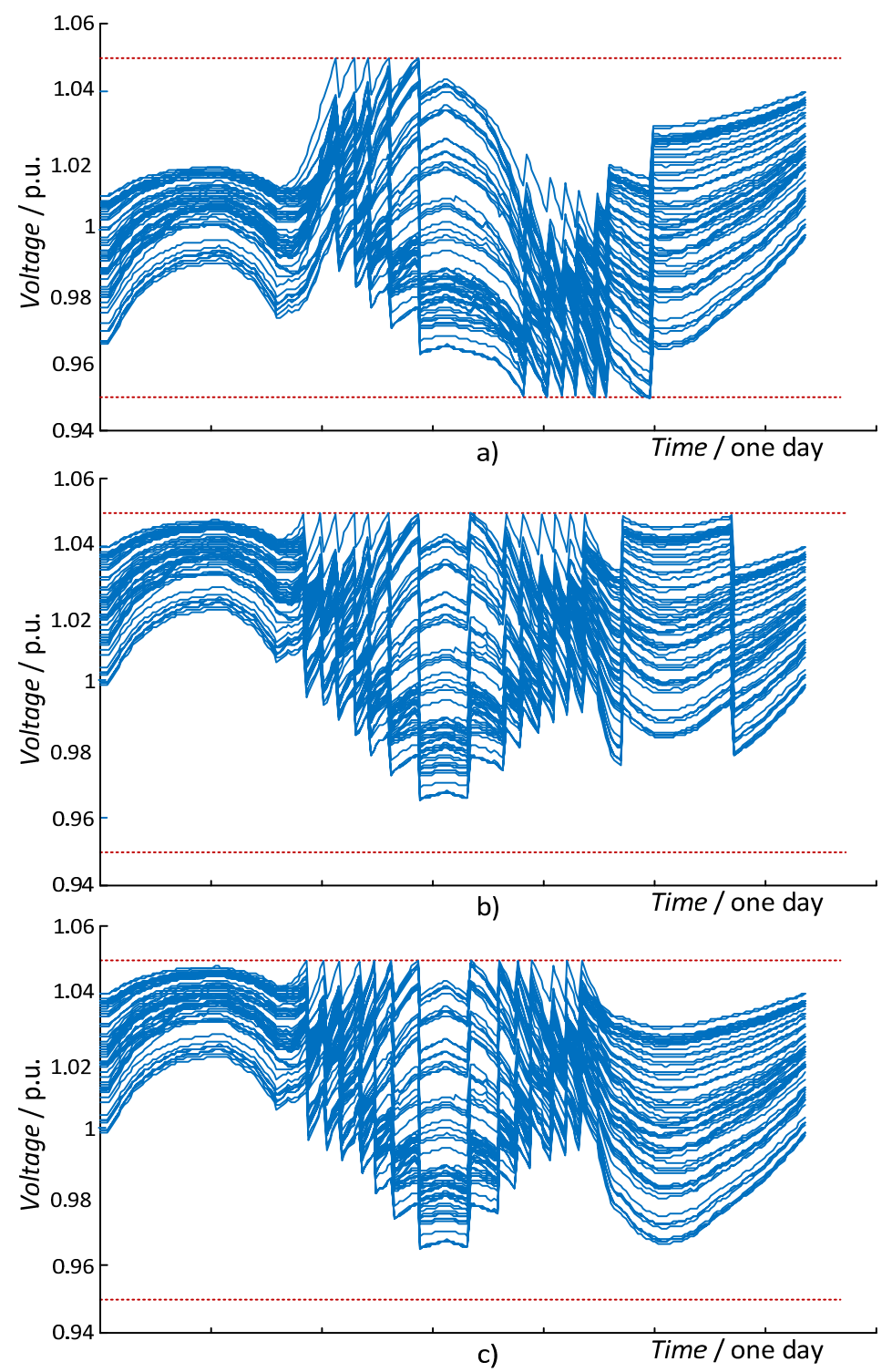


The results from Table 1 indicate that the introduction of DG can reduce the network losses by about $32 \%$ compared to operation with a constant power factor. This is due to the shorter transmission paths to the customers.

When using the $Q(U)$ characteristic, the tap-changer operations were reduced compared to the $\cos \varphi=1$ operation. Within the zone prescribed in [4], the losses can differ, but in general they display an increasing trend. It must be noted that the DG at the end of the feeder has a significantly higher contribution to the system parameters control.

In the case of the time-dependent power factor, the losses can be reduced by about $6.5 \%$ compared to the static $Q(U)$ characteristic. The simulations also show that a majority of the savings can be obtained by local control by adjusting to the desired DG set-point. Up to $40 \%$ of the savings fall to OLTC set-point. By identifying an optimal tg $\varphi$, losses can be reduced by $6.5 \%$. A further $2.6 \%$ reduction in losses could be obtained by setting the tap-changer to an optimal set-point. On the other hand, on optimizing the algorithm to obtain a minimal $\operatorname{tg} \varphi, 1.8 \%$ of savings can be achieved and an additional $2.8 \%$ of savings can be achieved by setting the tap-changer to an optimal set-point.

Table 1. Simulation results for loads with constant power characteristic.

\begin{tabular}{lccc}
\hline \multicolumn{1}{c}{ Control } & $\begin{array}{c}\text { Tap-changer } \\
\text { Operations }\end{array}$ & $\begin{array}{c}\text { Losses } \\
\mathbf{( k W h )}\end{array}$ & $\begin{array}{c}\text { Annual savings } \\
\text { compared to static } \\
\boldsymbol{Q}(\boldsymbol{U}) \text { control }(\boldsymbol{\epsilon} / \mathbf{y r})\end{array}$ \\
\hline Without DG [Figure 10(a)] & 3 & 37,170 & $/$ \\
Constant $\cos \varphi$ [Figure 10(b)] & 10 & 25,467 & 8,541 \\
Static $Q(U)$ characteristic from [4] [Figure 10(c)] & 8 & 25,935 & $/$ \\
Optimal tg $\varphi$ [Figure 13(a)] & 12 & 24,253 & 30,697 \\
Minimal tg $\varphi$ and optimal tap-changer position & 14 & 24,737 & 21,864 \\
Optimal tg $\varphi$ and optimal tap-changer position [Figure 13(b)] & 16 & 23,561 & 43,326 \\
Optimal tg $\varphi$ and optimal tap-changer position [Figure 13(c)] & 14 & 23,787 & 39,201 \\
\hline
\end{tabular}

Figure 13(c) also presents the decision-making of the centralized voltage control. During the evening, the tap-changer did not change its position [in contrast to Figure 13(b)] and hence, saved two switching operations but increased the losses by ca. $0.9 \%$. The algorithm can, on the basis of load forecast, determine the losses in advance and decide if the savings are sufficiently large to make two tap-changer operations or leave it in the current state.

Figure 14 presents how the feeder losses change as a function of uniform $\operatorname{tg} \varphi$. There is always one operation point where the losses are minimal. Simulation results have showed that savings obtained by setting an optimal tg $\varphi$ can achieve up to $90 \%$ of all the savings when using different load characteristics such as constant current or impedance. This implies that the characteristics of loads in the network have to be examined carefully.

Figure 15 displays optimal $\operatorname{tg} \varphi$ for one feeder for different types of loads. The red line and blue line represent the optimal $\operatorname{tg} \varphi$ with loads composed of $50 \%$ of constant power and $50 \%$ of constant impedance and $40 \%$ of constant power and $60 \%$ of constant impedance, respectively. 
Figure 14. Example of calculated feeder losses as a function of uniform $\operatorname{tg} \varphi$ for one operating point when consumption was high.

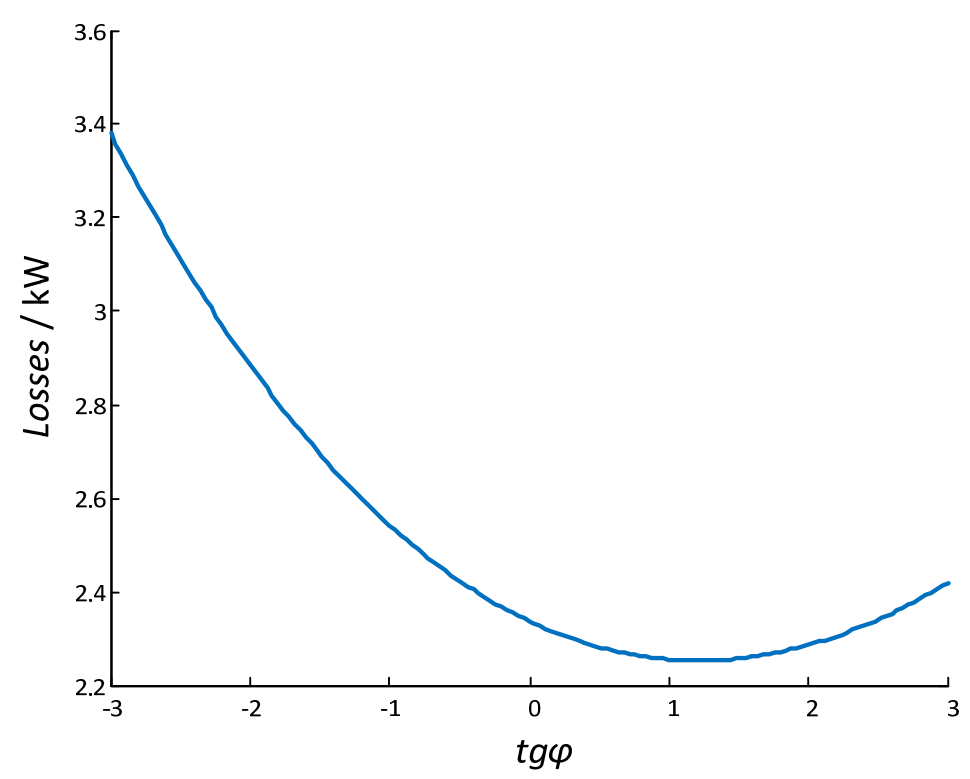

It is observed that in both the cases during strong solar radiation situations, the differences between the calculated $\operatorname{tg} \varphi$ are small. Towards the evening, the required $\operatorname{tg} \varphi$ increases greatly. This results from still high-energy consumption and minimal generation from photovoltaic plants, which represent the largest share of DG in the network. Therefore, the differences between the desired $\operatorname{tg} \varphi$ are slightly greater. The greater the share of DG, the less significant the load characteristic is.

Figure 15. Desired optimal tg $\varphi$ using different load characteristics.

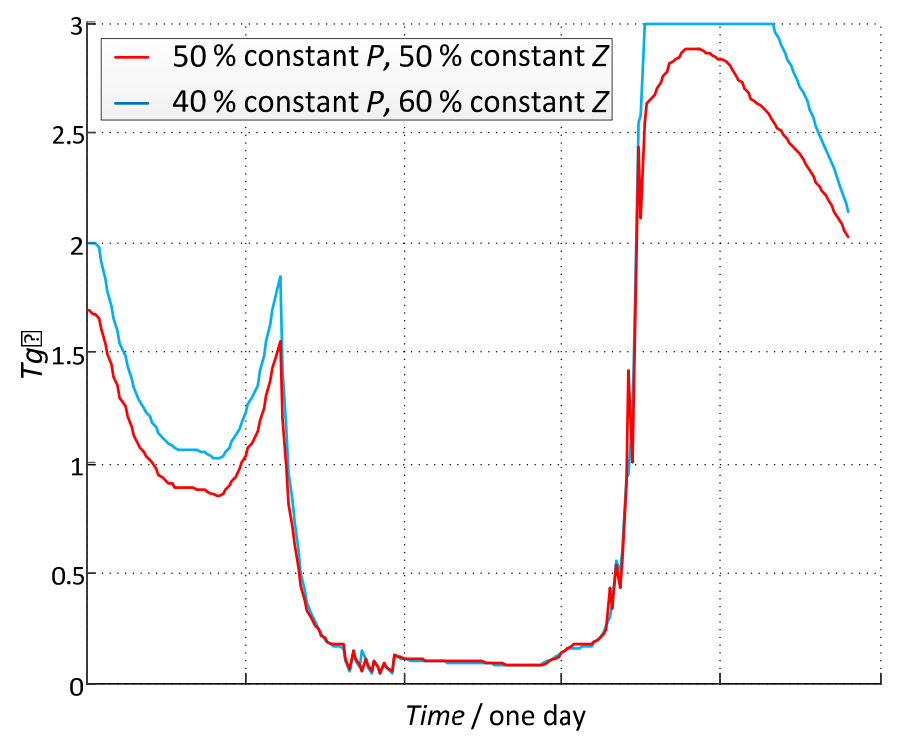

In summary, the presented coordinated control has several advantages. Investments in equipment maintenance and cost of the network operation can be minimized and a fair reactive power dispatch can be achieved. 


\subsection{DG Penetration Limits}

To achieve fairness regarding reactive power contributions, the amount of reactive power is not based on their electrical location on the feeder, but only on their size, which is one of the premises of this article. Their electrical location is limited only to one feeder as a whole unit. Although generators at the end have a higher effect on the voltage profile, they are also more burdened. To illustrate this, the voltage rise of one of the feeders is presented in Figure 16(a) and the optimal $\operatorname{tg} \varphi$ for this feeder is shown in Figure 16(b). To mitigate this voltage rise during the peak $h$, the generators have to change their trajectory and consume reactive power. The green line in Figure 16(b) represents the $\operatorname{tg} \varphi$ when all the generators contributed the same amount of reactive power and the blue line indicates instances when only a few of them at the end of the feeder were chosen to contribute reactive power. It can be seen that in the latter case, the generators have to operate with a $\operatorname{tg} \varphi<-1.7$, which is a very strict requirement. During the peak $\mathrm{h}$ when their active power is at a maximum, they usually have a low level of reactive power on reserve.

Figure 16. Different types of voltage control to mitigate the voltage rise. (a) One-day voltage profile of all MV/LV substations measured at one feeder; (b) optimal $\operatorname{tg} \varphi$ for one feeder with respect to voltage limits for all the generators (green line). Generators have to contribute more reactive power during peak $h$ if only a few of them at the end of the feeder are requested to contribute (blue line).
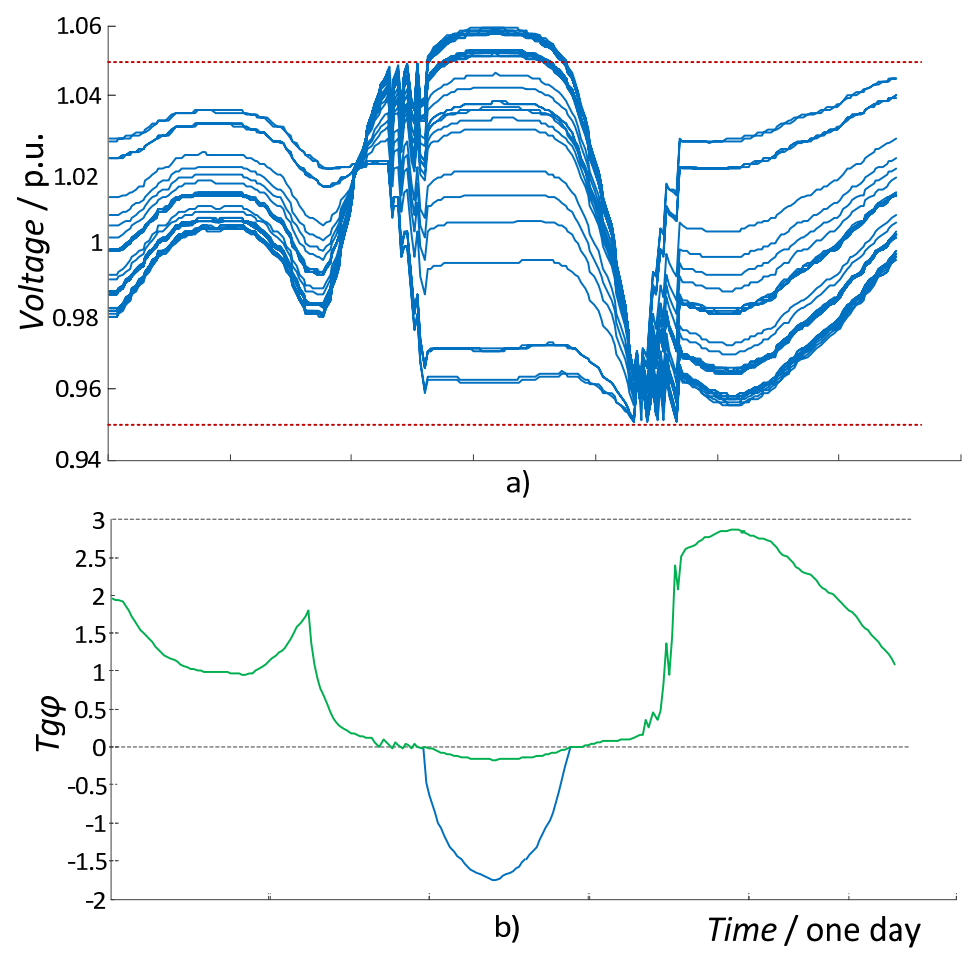

Figure 17(b) additionally shows the optimal $\operatorname{tg} \varphi$ for four different feeders. The red line represents the longest feeder, violet line represents a feeder with low DG share, blue line represents a feeder with a large amount of underground cables and green line represents an average feeder. It is observed that during the voltage rise of some feeders (red line), some other feeders have to raise the voltage so that the lower voltage limit is not reached. Figure 17(a) indicates the voltage profile for the described case. 
With a uniform $\operatorname{tg} \varphi$, up to $40 \%$ more DG can be integrated into the network compared to the static $Q(U)$ characteristic.

Figure 17. Voltage control to mitigate the voltage rise with a uniform $\operatorname{tg} \varphi$ for every feeder. (a) One-day voltage profile of MV/LV substations measured at the critical areas around the distribution system; (b) optimal tg $\varphi$ for four different feeders. As the penetration of DG around the twelfth hour is very high, $\operatorname{tg} \varphi$ has to withdraw its optimal daily trajectory.

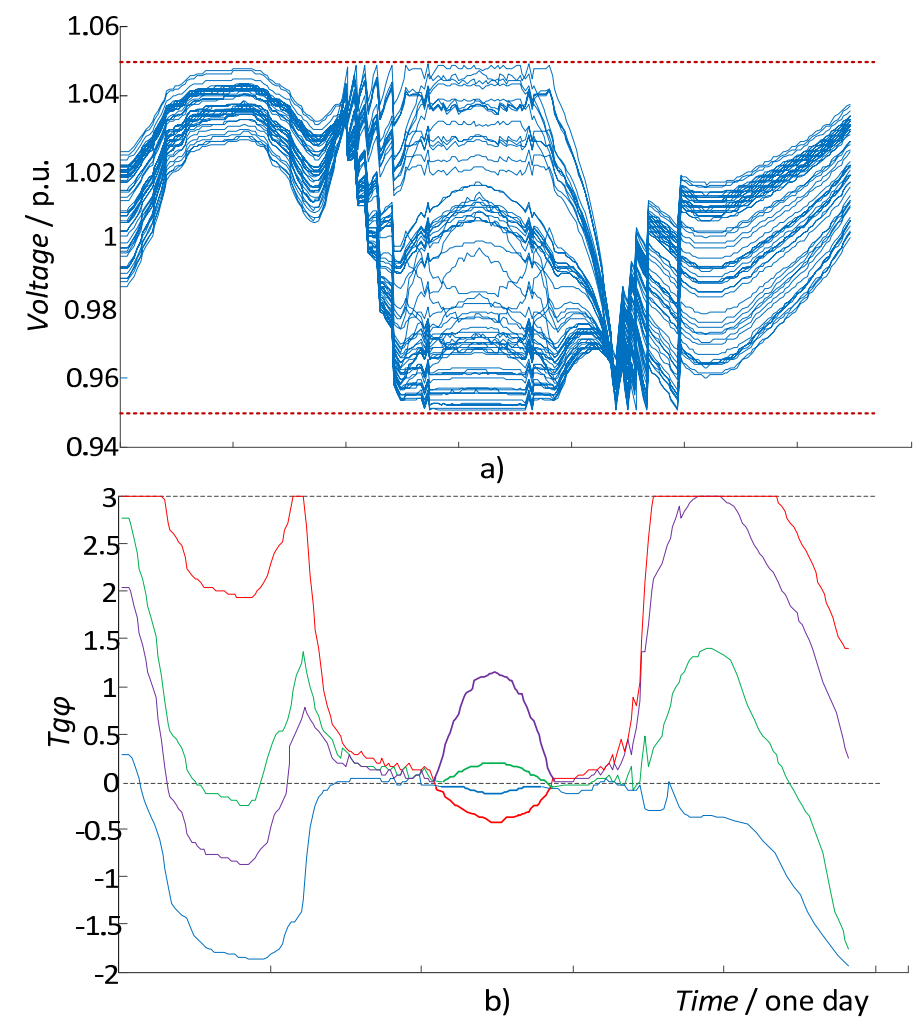

\section{Conclusions}

This article deals with the problem of line loss minimization in distribution networks with a high proportion of DG. As their share in the networks is increasing rapidly, "smart" and cost effective control solutions have to be developed to avoid costly network reinforcement due to voltage rises.

In this article a coordinated voltage-control algorithm has been presented for use in distribution networks with high distributed generation penetration. It is based on progress in recent technologies, which enable two-way communications and high processor power. The main purpose of this research was a desire to minimize line losses with respect to a fair contribution of reactive power of all the DG in the same feeder. As retail customers typically have no choice where they are located along the feeder, it seems unfair that they are required to produce or consume a large amount of reactive power and thus, bear all the burden and responsibility for the voltage rise along the entire feeder. This means that their contribution in ancillary services is not based by their electrical location on one feeder but is limited only on one feeder as a whole unit.

Local and centralized OLTC controls have been combined into one coordinated control whose parameters are calculated by a central computer system. The solution has been tested by means of computer simulations on a real medium-voltage Slovenian distribution network. 
The proposed method is simple in structure and easy to manipulate. Since the algorithm functions for many types of networks, minimizes investments and tries to equally spread the control burden, it represents a viable regulatory solution for the future Smart Grids.

As the practical implementation of the proposed algorithm seems viable, it will be thoroughly investigated and reported in our future work, with a focus on maximizing and facilitating DG integration.

\section{References}

1. Turitsyn, K.; Šulc, P.; Backhaus, S.; Chertkov, M. Options for control of reactive power by distributed photovoltaic generators. Proc. IEEE 2011, 99, 1063-1073.

2. Masters, C.L. Voltage rise: The big issue when connecting embedded generation to long $11 \mathrm{kV}$ overhead lines. Power Eng. J. 2002, 16, 5-12.

3. Blažič, B.; Pfajfar, T.; Papič, I. Voltage Control in Networks with Distributed Generation-A Case Study. In Proceedings of 2009 IEEE PES/IAS Conference on Sustainable Alternative Energy (SAE), Valencia, Spain, 28-30 September 2009; pp. 1-6.

4. SODO d.o.o. Navodila za Priključevanje in Obratovanje Elektrarn Inštalirane Moči do $10 \mathrm{MW}$. SODO d.o.o.: Maribor, Slovenia, 2010. Available online: http://www.sodo.si/_files/366/SONDO \%202011\%20Priloga\%205.pdf (accessed on 22 January 2012).

5. Kacejko, P.; Adamek, S.; Wydra, M. Optimal Voltage Control in Distribution Networks with Dispersed Generation. In Proceedings of Innovative Smart Grid Technologies Conference Europe (ISGT Europe), Gothenburg, Sweden, 11-13 October 2010; pp. 1-4.

6. Chessmore, D.T.; Lee, W.-L.; Muston, W.E.; Anthony, T.L.; Daniel, F.; Kohrmann, L. Voltage-Profile Estimation and Control of a Distribution Feeder. IEEE Trans. Ind. Appl. 2009, 45i, 1467-1474.

7. Bletterie, B.; Goršek, A.; Blažič, B.; Uljanić, B.; Brabandere, K.D.; Dierckxsens, C.; Fawzy, T.; Deprez, W.; Dexters, A. Innovative Control Concepts and Smart Planning for Active Operation of Distribution Networks With Large Amounts of PV Generation; Technical Report for MetaPV Project: University of Ljubljana, Ljubljana, Slovenia, 2011; pp. 96-100.

8. Brunner, H.; Lugmaier, A.; Bletterie, B.; Fechner, H.; Bründlinger, R. DG DemoNetz-Konzept; Österreichisches Forschungs und Prüfzentrum Arsenal: Wien, Austria, 2010.

9. Kovač, M. Davki; Zavod IRC: Ljubljana, Slovenia, 2010; pp. 7-9. Available online: http://www.impletum.zavod-irc.si/docs/Skriti_dokumenti/Davki-Kovac.PDF (accessed on 12 April 2012).

10. Austrian Energy Agency (AEA). European Smart Metering Landscape Report, Smart Regions Deliverable 2.1; AEA: Vienna, Austria, 2011.

11. European Commission, Joint Research Centre, Institute for Energy. Smart Grid Projects in Europe: Lessons Learned and Current Developments; Luxembourg: Publications Office of the European Union: Petten, The Netherlands, 2011.

12. Australian Trade Commission (Austrade). Australian Energy Efficiency Market Industry Capability Report. 2010. Clean Energy and Environment Team, Austrade: Sydney, Australia, July 2010. 
13. He, Y.; Jenkins, N.; Wu, J.; Eltayeb, M. ICT Infrastructure for Smart Distribution Networks. In Proceedings of the 2010 IEEE International Symposium on Power Line Communications and Its Applications (ISPLC), Rio de Janeiro, Brazil, 28-31 March 2010; pp. 319-324.

14. Tornqvist, B.; Fontela, M.; Mellstrand, P.; Gustavsson, R.; Andrieu, C.; Bacha, S.; Hadjsaid, N.; Besanger, Y. Overview of ICT Components and Its Application in Electric Power Systems. In Proceedings of the 2nd International Conference on Critical Infrastructures (CRIS 2004), Grenoble, France, October 2004.

15. Liu, Y.; Bebic, J.; Kroposki, B.; Bedout, J.D.; Ren, W. Distribution System Voltage Performance Analysis for High-Penetration PV. In Proceedings of IEEE Energy2030, Atlanta, GA, USA, 2008; pp. 1-8.

16. Kundur, P. Power System Stability and Control; McGraw-Hill: New York, NY, USA, 2009.

17. Lorenz, E.; Hurka, J.; Heinemann D.; Beyer, H.G. Irradiance forecasting for the power prediction of grid-connected photovoltaic systems. IEEE J. Sel. Top. Appl. Earth Obs. Remote Sens. 2009, 2 , 2-10.

18. Giebel, G.; Landberg, L.; Kariniotakis G.; Brownsword, R. State-of-the Art on Methods and Software Tools for Short-Term Prediction of Wind Energy Production. In Proceedings of European Wind Energy Conference \& Exhibition, Madrid, Spain, 2003.

19. Yanguas-Peña, A.; Mendoza-Villena, M.; Falces-De Andrés, A.; Lara-Santillán, P.; García-Garrido, E.; Zorzano-Alba, E. Short Term Load Forecasting For Low-Voltage Substations Using Genetically Optimized Fuzzy Inference Systems. In Proceedings of 11th Spanish-Portuguese Congress in Electrical Engineering, Zaragoza, Spain, 2009.

20. Lourenço, J.M.; Santos, P.J. Short Term Load Forecasting Using Gaussian Process Models. In Proceedings of Instituto de Engenharia de Sistemas e Computadores de Coimbra, Coimbra, Portugal, 2010.

21. Firat, U.; Engin, S.N.; Saraclar, M.; Ertuzun, A.B. Wind Speed Forecasting Based on Second Order Blind Identification and Autoregressive Model. In Proceedings of Ninth International Conference on Machine Learning and Applications, Washington, DC, USA, 2010; pp. 686-691.

22. Kang, M.C.; Sohn, J.M.; Park, J.Y.; Lee, S.K.; Yoon, Y.T. Development of Algorithm for Day Ahead PV Generation Forecasting Using Data Mining Method. In Proceedings of the IEEE 54th International Midwest Symposium on Circuits and Systems (MWSCAS), Seoul, Korea, 7-10 August 2011; pp. 1-4.

23. Cococcioni, M.; D'Andrea, E.; Lazzerini, B. 24-hour-ahead forecasting of energy production in solar PV systems. In Proceedings of the 11th International Conference on Intelligent Systems Design and Applications (ISDA), Pisa, Italy, 22-24 November 2011; pp. 1276-1281.

24. Zimmerman, R.D.; Murillo-Sánchez, C.E.; Thomas, R.J. MATPOWER Steady-State Operations. Planning and Analysis Tools for Power Systems Research and Education. IEEE Trans. Power Syst. 2011, 26, 12-19.

(C) 2012 by the authors; licensee MDPI, Basel, Switzerland. This article is an open access article distributed under the terms and conditions of the Creative Commons Attribution license (http://creativecommons.org/licenses/by/3.0/). 